\title{
Intoxication chronique à l'arsenic
}

\section{Chronic arsenic poisoning}

\section{Anne LE BOUIL ${ }^{*(1)}$, Martine AVENEL-AUDRAN ${ }^{(2)}$, Anne-Marie BOURGEAIS ${ }^{(2)}$, Jean-Luc VERRET ${ }^{(2)}$, Pierre ALLAIN ${ }^{(1)}$}

(1) Laboratoire de Pharmacologie et Toxicologie, CHU - 49033 ANGERS Cedex 01 (2) Service de Dermatologie, CHU - 49033 ANGERS Cedex 01

* Auteur à qui adresser la correspondance : Anne LE BOUIL, Laboratoire de Pharmacologie et Toxicologie, CHU, 4, rue Larrey - 49033 ANGERS Cedex 01 - Tél : 33 (0)2 41353644 - Fax : 33 (0)2 41354877

(Reçu le 5 avril 2001 ; accepté le 18 mai 2001)

\section{RÉSUMÉ}

Un homme de 75 ans est hospitalisé en Dermatologie pour une pigmentation cutanée diffuse du tronc avec des petites taches achromiques et des papules kératosiques palmaires dans un contexte d'altération de l'état général. Le diagnostic clinique d'arsenicisme chronique est confirmé par des dosages d'arsenic (arsenic inorganique et métabolites) dans le plasma, l'urine et les phanères et ceci par spectrophotométrie d'absorption atomique avec four graphite et correction Zeeman (GFAAS). L'acide monométhylarsonique (MMA) et l'acide diméthylarsinique (DMA) sont dosés dans l'urine par chromatographie liquide couplée à la spectrométrie de masse en tandem avec ionisation par électrospray (LC-ES-MS-MS). La consommation d'un vin fabriqué par le patient est à l'origine de l'intoxication. Une concentration élevée d'arsenic y est retrouvée ( $7 \mathrm{mg} / \mathrm{l})$. Il semblerait qu'une erreur dans la manipulation d'un fongicide à l'arsénite de sodium utilisé pour le traitement de la vigne, le Pyralesca ${ }^{\circledR}$, soit la cause de la contamination du vin.

\section{MOTS-CLÉS}

Intoxication chronique, arsenic, LC-ES-MS-MS, GFAAS.

\section{SUMMARY}

A 75-year old man was hospitalized in the dermatology unit for cutaneous manifestations consisting of diffuse hyperpigmentation with scattered white spots on the trunk and palmoplantar keratosis. The determination of arsenic (inorganic arsenic and metabolites) by graphite furnace atomic absorption spectrometry (GFAAS) in plasma, urine, nail and hair confirmed the diagnosis of chronic arsenic poisoning. The monomethylarsonic acid (MMA) and the dimethylarsinic acid (DMA) were measured in urine by liquid chromatography-tandem mass spectrometry with electrospray ionization (LC-ES-MS-MS). The intoxication was due to consumption of a home-made wine. The arsenic concentration in the wine was $7 \mathrm{mg} / \mathrm{l}$. A mistake in the handling of Pyralesca ${ }^{\circledR}$, an arsenite-based fungicide used for the treatment of the vine, could explain the contamination of the wine.

\section{KEY-WORDS}

Chronic intoxication, arsenic, LC-ES-MS-MS, GFAAS. 


\section{Introduction}

Les intoxications chroniques à l'arsenic sont essentiellement d'origine professionnelle (métallurgie, industrie du verre, des pigments, préservation du bois) ou liées à l'environnement. Dans ce dernier cas, il s'agit de l'hydroarsenicisme chronique régional endémique observé dans certains pays d'Amérique (1) ou à Taïwan (2).

La symptomatologie de l'intoxication chronique à l'arsenic associe troubles digestifs (nausées, vomissements), mélanodermie avec kératose palmo-plantaire, polynévrite des membres inférieurs, hypertension portale, troubles hématologiques. L'exposition à l'arsenic est associée à une incidence accrue de cancers bronchiques par inhalation et de cancers de la peau et d'organes internes par ingestion (3).

L'arsenicisme chronique est devenu une maladie rare et en dehors d'un contexte d'exposition professionnelle, il faut toujours rechercher la source de l'arsenic en cause.

\section{Observation}

Un homme de 75 ans, ancien ouvrier viticulteur, est hospitalisé en Dermatologie pour une pigmentation cutanée diffuse avec des petites taches achromiques du tronc et des papules kératosiques palmaires dans un contexte d'altération de l'état général (4). La symptomatologie évoque l'intoxication chronique à l'arsenic. Le patient est déjà rentré chez lui quand les résultats des dosages d'arsenic dans le plasma, l'urine et les phanères confirment le diagnostic clinique. Le patient est revu en consultation externe un mois après l'hospitalisation. De nouveaux prélèvements de sang et d'urine sont alors réalisés. L'entretien avec le patient permet d'apprendre que celui-ci consomme par jour environ un à deux litres de vin rouge fabriqué par lui-même. L'analyse d'un échantillon du vin montre une concentration très élevée d'arsenic. Le produit à l'origine de la contamination serait le Pyralesca ${ }^{\circledR}$, fongicide à base d'arsénite de sodium, utilisé pour le traitement d'hiver de la vigne. Les circonstances de cette contamination ne sont pas élucidées : mauvaise utilisation du produit ?

Il est fortement recommandé au patient de ne plus consommer son vin.

Un suivi du patient avec des contrôles de l'arsenic plasmatique et urinaire est mis en place.

\section{Matériel et Méthode}

\section{Dosage de l'arsenic dans l'urine}

La préparation de l'échantillon, selon une technique déjà décrite (5), consiste en une extraction par le chlo- roforme permettant d'éliminer l'arsénobétaïne.

L'extrait ainsi obtenu est analysé :

- par GFAAS (5) : l'arsenic dosé correspond à l'arsenic inorganique (Asi) et ses métabolites c'est à dire la somme Asi + MMA + DMA.

- par LC-ES-MS-MS (6, 7): les concentrations de MMA et de DMA sont alors mesurées.

\section{Dosage de l'arsenic dans le plasma}

Après une précipitation des protéines par l'acide sulfosalicylique, l'échantillon subit la même préparation que l'urine avant d'être analysé par GFAAS. La concentration d'arsenic mesurée correspond à la somme $\mathrm{As}_{\mathrm{i}}+$ MMA + DMA.

\section{Dosage de l'arsenic dans les phanères}

Les cheveux ou les ongles sont lavés par du dichlorométhane puis de l'eau déminéralisée et ensuite séchés à l'étuve à $37^{\circ} \mathrm{C}$. Environ $100 \mathrm{mg}$ de cheveux ou d'ongles lavés, sont digérés par $1 \mathrm{ml}$ d'acide nitrique $(65 \%$ Suprapur) pendant 48 heures à température ambiante suivies de 2 heures à $70^{\circ} \mathrm{C}$. Le volume est ensuite complété à $5 \mathrm{ml}$ par de l'eau déminéralisée. Cette solution est analysée par GFAAS. La concentration d'arsenic mesurée correspond à l'arsenic total.

\section{Dosage de l'arsenic dans le vin}

A $1 \mathrm{ml}$ de vin est ajouté $1 \mathrm{ml}$ d'acide nitrique $(65 \%$ Suprapur). Le mélange est laissé à température ambiante pendant une nuit puis à $70^{\circ} \mathrm{C}$ pendant 2 heures. Le volume est complété à $10 \mathrm{ml}$ par de l'eau déminéralisée. Cette solution est analysée par GFAAS. La concentration d'arsenic mesurée correspond à l'arsenic total.

\section{Résultats et Discussion}

Les résultats des dosages effectués dans les milieux biologiques sont présentés dans le tableau I.

Les concentrations d'arsenic dans les premiers prélèvements, effectués lors de l'hospitalisation, sont très supérieures aux valeurs normales et ceci quel que soit le milieu. Ces prélèvements ont été réalisés alors que le patient était hospitalisé depuis quatre jours et donc soustrait à de nouveaux apports d'arsenic. Dans ce premier échantillon urinaire, l'arsenic se trouve principalement sous forme organique : $20,4 \%$ sous forme de MMA et $77,3 \%$ sous forme de DMA. Ces résultats sont en accord avec ceux décrits dans la littérature. En effet, Mahieu et col.(8), dans un cas d'intoxication aiguë par l'anhydride arsénieux, rapportaient que la proportion d'arsenic urinaire excrété sous forme organique atteignait $90 \%$ à partir du $5^{\text {cme }}$ jour. Tam et col.(9), après administration orale de $0,01 \mu \mathrm{g} \mathrm{d}^{174} \mathrm{As}$ sous forme inorganique chez six volontaires, mon- 
Tableau I : Concentrations d'arsenic dans les milieux biologiques.

\begin{tabular}{|c|c|c|c|c|c|c|}
\hline $\begin{array}{l}\text { Date } \\
\text { situation }\end{array}$ & $\begin{array}{l}\text { plasma } \\
\mathrm{As}_{\mathrm{i}}+\mathrm{MMA}+\mathrm{DMA} \\
\mathrm{N}<10 \mu \mathrm{g} / \mathrm{l}\end{array}$ & $\begin{array}{l}\text { urine } \\
A s_{\mathrm{j}}+\mathrm{MMA}+\mathrm{DMA} \\
\mathrm{N}<30 \mu \mathrm{g} / \text { gcréat. }\end{array}$ & $\begin{array}{l}\text { MMA } \\
\text { ( } \mu \mathrm{g} / \mathrm{gcré} a t .)\end{array}$ & $\begin{array}{l}\text { DMA } \\
(\mu \mathrm{g} / \text { gcréat. })\end{array}$ & $\begin{array}{l}\text { cheveux } \\
\mathrm{As}_{\text {total }} \\
\mathrm{N}<1 \mu \mathrm{g} / \mathrm{g}\end{array}$ & $\begin{array}{l}\text { ongles } \\
\begin{array}{l}\mathrm{As} \\
\mathrm{N}<1,5 \mu \mathrm{g} / \mathrm{g}\end{array}\end{array}$ \\
\hline $\begin{array}{l}\text { 15/09/97 } \\
\text { hôpital }\end{array}$ & 26 & 1772 & 361 & 1369 & 80 & 120 \\
\hline $\begin{array}{l}\text { 17/10/97 } \\
\text { domicile }\end{array}$ & 60 & 4268 & 978 & 2237 & & \\
\hline $\begin{array}{l}02 / 12 / 97 * \\
\text { domicile }\end{array}$ & $<10$ & 480 & & & & \\
\hline $\begin{array}{l}\text { 14/10/98 } \\
\text { hôpital }\end{array}$ & $<10$ & 62 & & & & \\
\hline $\begin{array}{l}\text { 09/11/98 } \\
\text { domicile }\end{array}$ & $<10$ & 21 & & & & \\
\hline
\end{tabular}

* cinq semaines après l'arrêt théorique de consommation du vin

traient qu'au $5^{\text {ime }}$ jour 71,3\% de l'arsenic présent dans l'urine était sous forme de DMA. Des résultats similaires étaient obtenus par Buchet et col. (10) après administration d'une dose unique d'arsénite de sodium chez des volontaires.

Des prélèvements d'urine et de sang ont été réalisés un mois plus tard au cours d'une consultation. Entre l'hospitalisation et cette consultation, le patient a continué d'être exposé, l'origine de l'arsenic en cause n'étant pas encore connue. Ceci se traduit par des concentrations d'arsenic plasmatique et urinaire plus élevées que celles observées lors de l'hospitalisation. Les proportions relatives des différentes formes d'arsenic mesurées dans l'urine : $24,7 \%$ d'As $_{\mathrm{i}}, 22,9 \%$ de MMA et $52,4 \%$ de DMA reflètent un apport continu et sont comparables à celles observées chez des sujets exposés professionnellement et chez des sujets témoins (11).

Les concentrations d'arsenic dans les prélèvements de contrôle, effectués après avoir recommandé au patient de ne plus consommer son vin, sont toujours inférieures à la limite de quantification dans le plasma. Cependant, les résultats obtenus dans l'urine laissent à penser, qu'au début la recommandation n'était peut être pas toujours suivie.

La concentration d'arsenic mesurée dans le vin, $7 \mathrm{mg} / \mathrm{l}$, est très supérieure à la limite maximale admissible de 0,2 mg/l (1998, Office International de la Vigne et du Vin). D'autres vins de la région, quatre "Anjou rouge" de producteurs différents, ont également été analysés : leur concentration en arsenic était inférieure à $50 \mu \mathrm{g} / \mathrm{l}$. La teneur excessive en arsenic associée à une consommation importante (1 à 2 litres de vin/jour) expliquent l'intoxication. La façon dont le patient a contaminé son vin reste cependant non élucidée. En effet, le
Pyralesca®, fongicide à base d'arsénite utilisé par le patient, a comme indication le traitement d'hiver de la vigne donc très à distance de la vendange.

Cette observation est assez similaire à celle décrite par Franzblau et col. en 1989 (12). Il s'agissait d'un couple nord-américain intoxiqué par l'eau de leur puits. La concentration d'arsenic dans l'eau était de $10 \mathrm{mg} / \mathrm{l}$ (la valeur maximale recommandée en France est $50 \mu \mathrm{g} / \mathrm{l}$ et passera bientôt à $10 \mu \mathrm{g} / \mathrm{l}$ comme aux USA). Les concentrations d'arsenic dans des prélèvements biologiques réalisés quelques jours après l'arrêt de la consommation de l'eau contaminée étaient les suivantes : 2260 $\mu \mathrm{g} / \mathrm{l}$ d'urine chez la femme et $488 \mu \mathrm{g} / \mathrm{l}$ d'urine, $61 \mu \mathrm{g} / \mathrm{l}$ de sang, 32 à $52 \mu \mathrm{g} / \mathrm{g}$ de cheveux chez l'homme.

\section{Conclusion}

Après la confirmation du diagnostic d'arsenicisme chronique par des dosages d'arsenic dans le plasma, l'urine et les phanères, la recherche de la source d'arsenic responsable de l'intoxication est essentielle afin d'éviter les contaminations ultérieures.

Dans cette observation, les habitudes de vie du patient ont facilité cette démarche. Le Pyralesca ${ }^{\circledR}$, fongicide utilisé pour le traitement d'hiver de la vigne, est fort vraisemblablement à l'origine de la contamination du vin que fabriquait et consommait le patient.

Actuellement, les dérivés de l'arsenic encore utilisés comme produits phytosanitaires sont l'arsénite de sodium (Pyralesca ${ }^{\circledR}$, Pyral Rep ${ }^{\circledR}$, Pyral Rep Fort ${ }^{\circledR}$, Pyralumnol $2000^{\circledR}$ ) et le diméthylarsinate de sodium encore appelé cacodylate de soude. Ce dernier produit, utilisé comme antifourmis, est très peu toxique. 


\section{Références}

1. Cebrian M.E., Albores A., Aguilar M., Blakely E.,Chronic arsenic poisoning in the north of Mexico. Human Toxicol. $1983 ; 2: 121-33$.

2. Hsueh Y. M., Huang Y. L., Huang C. C., Chen H. M., Yang M. H., Lue L. C., Chen C.J., Urinary levels of inorganic and organic arsenic metabolites among residents in an arseniasis-hyperendemic area in Taïwan. J. Toxicol. Environ. Health PT A. 1998 ; 54 : 431-44.

3. Bates M. N., Smith A. H., Hopenhayn-Rich C., Arsenic ingestion and internal cancers : a review. Am. J. Epidemiol. 1992 ; 135 : 462-76.

4. Bourgeais A-M., Avenel-Audran M., Le Bouil A., Bouyx C., Allain P., Verret J.L., Arsenicisme chronique. Ann. Dermatol. Venereol. $2001 ; 128$ : 527-30.

5. Le Bouil A., Notelet S., Cailleux A., Turcant A., Allain P., Dosage de l'arsenic urinaire après séparation de l'arsénobétaïne. Toxicorama, $1997 ; 9: 171-6$.

6. Le Bouil A., Cailleux A., Turcant A., Allain P., Chromatographie liquide couplée à la spectrométrie de masse en tandem : application au dosage des formes organiques de l'arsenic dans l'urine. Toxicorama, 1999 ; $11: 140-6$.

7. Le Bouil A, Cailleux A., Turcant A., Allain P., Determination of monomethylarsonic acid and dimethylarsinic acid in urine by liquid chromatography-tandem mass spectrometry. J. Anal. Toxicol. 1999 ; 23 : 257-61.
8. Mahieu P., Buchet J. P., Lauwerys R., Evolution clinique et biologique d'une intoxication orale aiguë par l'anhydride arsénieux et considérations sur l'attitude thérapeutique. Journal de Toxicologie Clinique et Expérimentale, $1987 ; 4: 273-8$.

9. Tam G. K. H., Charbonneau S. M., Bryce F., Pomroy C., Dandi E., Metabolism of inorganic arsenic (74As) in humans following oral ingestion. Toxicol. Appl. Pharmacol. $1979 ; 50: 319-22$.

10. Buchet J. P., Lauwerys R., Roels H., Comparison of the urinary excretion of arsenic metabolites after a single oral dose of sodium arsenite, monomethylarsonate, or dimethylarsinate in man. Int. Arch. Occup. Environ. Health. $1981 ; 48: 71-9$.

11. Hopenhayn-Rich C., Smith A. H., Goeden H. M., Human studies do not support the methylation threshold hypothesis for toxicity of inorganic arsenic. Environ. Res., $1993 ; 60: 161-77$.

12. Franzblau A., Lilis R., Acute arsenic intoxication from environmental arsenic exposure. Arch. Environ. Health, $1989 ; 44: 385-90$. 